\title{
1 Die stationäre Notfallversorgung in Deutschland
}

\author{
Anja Hohmann und David Herr
}

Die Notfallversorgung in den Notaufnahmen der Krankenhäuser ist neben der ambulanten kassenärztlichen Notfallbehandlung und dem Rettungsdienst Bestandteil des gegenwärtig dreigliedrigen Systems der medizinischen Notfallversorgung in Deutschland. Unterschiedliche Strukturen und Zuständigkeiten der einzelnen Akteure sollen hierbei im Sinne einer bedarfsgerechten Versorgung dazu dienen, den medizinischen und pflegerischen Aufwand an die Dringlichkeit des Notfalls anzupassen und Kapazitäten für schwere Fälle vorzuhalten.

Im Zusammenhang mit steigenden Inanspruchnahmen von Leistungen vor allem der Notaufnahmen sowie des Rettungsdienstes haben in den vergangenen Jahren diverse Akteure Stellungnahmen und Analysen zum Stand und zur Zukunft der Notfallversorgung in Deutschland veröffentlicht (vgl. SVR 2018). Der vorliegende Beitrag stellt die Strukturen und die empirische Lage zur Inanspruchnahme insbesondere der Krankenhaus-Notfallversorgung dar. Aktuell geplante Reformen im Bereich der Notfallversorgung sind nicht Gegenstand.

Die zentrale Aufgabe der Notaufnahmen der Krankenhäuser ist die Erstversorgung von „Gefahren für Leib und Leben“ sowie die Abklärung der Notwendigkeit einer stationären Behandlung (vgl. auch Urteil des Bundessozialgerichts von 2012). Sie versorgen Patientinnen und Patienten aus dem Rettungsdienst weiter, sind aber auch direkte Anlaufstelle für sie. Denn prinzipiell sind alle Akteure der Notfallversorgung verpflichtet, alle Patienten, die sich im Notfall an sie wenden, zu versorgen. So ist eine Notaufnahme auch für die Erstversorgung derjenigen, die von einem niedergelassenen Arzt hätten behandelt werden können, zuständig, wenn sich diese dort notfallmäßig vorstellen. 
Die subjektive Beurteilung durch die Patientinnen und Patienten, wann ein notfallmäßiger Behandlungsbedarf vorliegt, kann sich deutlich unterscheiden. Seitens notfallmedizinischer Fachgesellschaften wird der Begriff des medizinischen Notfalls zunächst allgemein als jede Veränderung des Gesundheitszustandes, für die der Betroffene oder eine Drittperson unverzügliche medizinische Versorgung als notwendig erachtet, definiert (Behringer et al. 2013). Werden Patienten, die sich selbstständig in der Notaufnahme vorstellen, zur Einschätzung dieser subjektiven Dringlichkeit befragt, zeigt sich, dass dies unter anderem vom Alter, Bildungsgrad und Geschlecht abhängig ist. Es zeigt sich auch, dass ein nicht unerheblicher Anteil der sich eigenständig vorstellenden Patienten ihre Behandlungsdringlichkeit als niedrig einstuft, also auch aus eigener Sicht nicht der Definition eines medizinischen Notfalls entspricht (Scherer et al. 2017).

Die Ergänzung bzw. teilweise Verschiebung des tatsächlichen Tätigkeitsspektrums der Krankenhaus-Notaufnahmen von der Notfallversorgung im engeren Sinn hin zu einer Akutversorgung ohne besondere Dringlichkeit bindet Ressourcen, was auch unter dem Aspekt der Versorgungsqualität ( $\mathrm{Pa}$ tientensicherheit) als problematisch anzusehen ist: So zeigen internationale Studien, dass überfüllte Notaufnahmen mit einer höheren Mortalitätsrate der aufgenommenen bzw. ambulant gesehenen Patienten assoziiert sind. Überdies gehen überfüllte Notaufnahmen mit einer größeren Anzahl von Patienten einher, die die Notaufnahmen aus eigenem Entschluss wieder verlassen, ohne von einem Arzt gesehen worden zu sein (Carter et al. 2014; Morley et al. 2018).

Diese zunehmend beobachtete Fehlinanspruchnahme in der Notfallversorgung ist ein Phänomen, welches nicht nur in Deutschland, sondern auch in verschiedenen inner- und außereuropäischen Gesundheitssystemen diskutiert wird. Jedoch zeigen OECD-Daten, dass das Wachstum der Inanspruchnahme von Notaufnahmen in Deutschland auch im internationalen Vergleich überdurchschnittlich hoch ist (Berchet 2015) - auch wenn die absoluten Fallzahlen der ambulanten Notfälle noch unter dem Durchschnitt anderer europäischer Länder liegen.

\subsection{Zeitliche Entwicklung der Anzahl der Notaufnahmepatienten}

\subsubsection{Stationäre Notfallversorgung}

Genaue Daten, wie viele deutsche Krankenhäuser eine Notaufnahme betreiben, gibt es, auch mangels einer einheitlichen baulichen und organisatorischen Definition, nicht. Schätzungen zufolge haben ca. zwei Drittel der 1.592 Allgemeinkrankenhäuser eine Notaufnahme (Geissler et al. 2017). Hierbei kann jedoch ein Krankenhaus mehrere Notaufnahmen betreiben, sodass für das Jahr 2015 ambulante Abrechnungsdaten aus 2.480 Notaufnahmen vorlagen - und somit deutlich mehr als die Gesamtzahl aller Krankenhäuser, auch wenn neben Allgemeinkrankenhäusern psychiatrische Schwerpunktkrankenhäuser mitgezählt werden (Stillfried et al. 2017).

Die stationären Fälle mit Aufnahmeanlass „Notfall“ machten im Jahr 2018 46,1\% der Krankenhausfälle aus, das entspricht ca. 8,6 Mio. Fällen (Spindler 2020). Diese Anzahl ist fast so hoch wie diejenige der Fälle mit Aufnahmeanlass „Einweisung durch Arzt“ (46,4\%; 8,7 Mio.). Die restlichen Aufnahmeanlässe verteilen sich auf Geburt und Verlegung sowie - verschwindend 


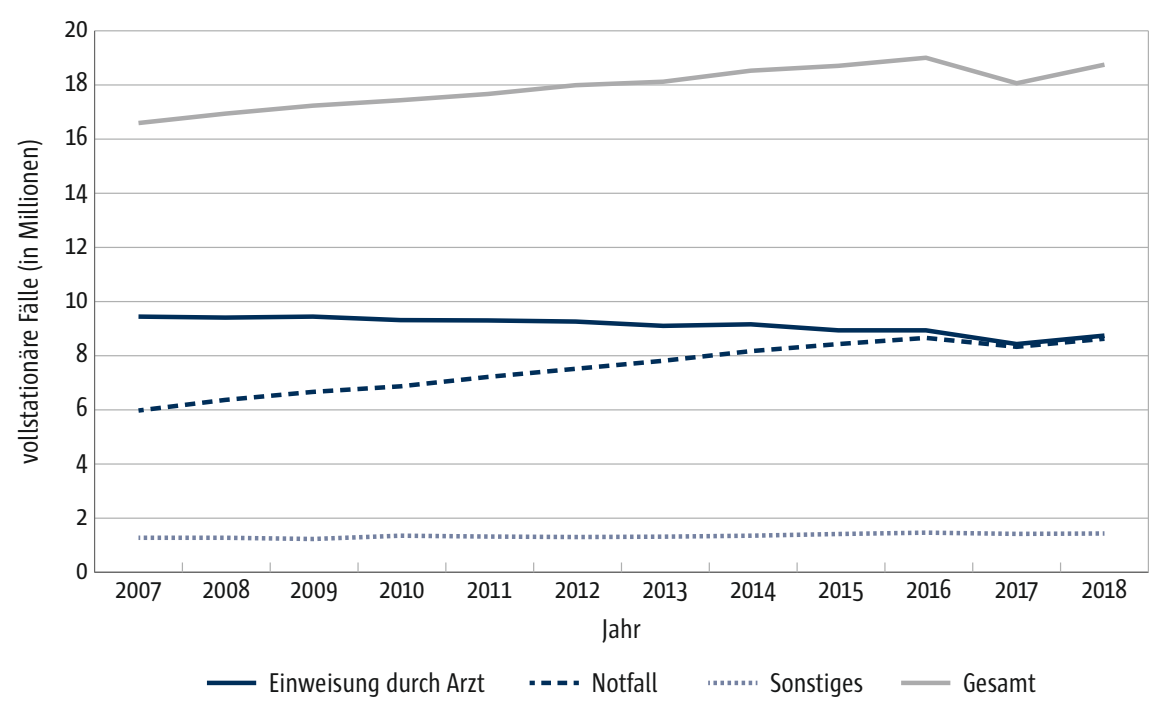

Abb. 1 Entwicklung vollstationärer Fälle nach Aufnahmeanlass. Die Kategorie „Sonstiges“ enthält Fälle mit Aufnahmeanlass „Geburt“, „Verlegung aus einem anderen Krankenhaus“ und „Aufnahme nach Reha-Behandlung“ (eigene Darstellung, ergänzt und modifiziert nach SVR 2018 auf Basis von Destatis 2019).

gering - Aufnahmen nach einer Reha. Die „Notfälle“ sind in den 10 Jahren zuvor deutlich angestiegen von ca. 6 Mio. Fällen im Jahr 2007 (s. Abb. 1). Seitdem ist trotz jüngstem Rückgang insgesamt eine deutliche Gesamt-Fallzahlsteigerung zu verzeichnen. Da die Aufnahmen durch Einweisung leicht rückläufig gewesen sind, erklärt der Gesamtanstieg sich vollständig aus den Notfällen. Bei diesen Zahlen handelt es sich allerdings um „administrative“ Notfälle.

\section{Administrative Notfälle versus medizinische Notfälle}

Die Krankenhausfälle, die in der Statistik mit Aufnahmeanlass „Notfall“ gezählt werden, entsprechen nicht zwingend der medizinischen Beurteilung eines Notfalls. Eine Aufnahme wird als „Notfall“ kodiert, wenn der Patient ohne Krankenhaus-Einweisung aufgenommen wird, wobei ein administrativer Spielraum besteht. Beispielsweise werden nur zwei Drittel der Aufnahmen mit den Diagnosen „Schlaganfall“ oder „Herzinfarkt" als administrativer Notfall kodiert. Manche Schlaganfallpatienten werden von niedergelassenen Ärzten dringlich eingewiesen und zählen dann nicht in diese Statistik. Umgekehrt werden auch Patienten von den Notaufnahmen aufgenommen, die potenziell auch zur elektiven Aufnahme hätten wiederkehren können. Die sogenannten "charakteristischen stationären Notfälle" (SVR 2018) sind zwischen 2007 und 2012 jährlich nur um knapp 2\% angestiegen (Schreyögg et al. 2014), die administrativen Notfälle hingegen jährlich um 4,4\%. 
Der Anstieg an administrativen Notfällen kann auch auf ein unzureichendes Versorgungsangebot im niedergelassenen Sektor hindeuten und wäre dann so zu interpretieren, dass (unabhängig von den tatsächlichen Diagnosen) weniger Patienten mit Einweisung eines ambulanten Arztes in die Notaufnahme kommen. So zeigt sich ein Zusammenhang zwischen dem regionalen Versorgungsangebot und stationären notfallmäßigen Aufnahmen: Die regionale Häufigkeit, mit der Patienten kurzzeitig - für maximal drei Tage - als Notfälle stationär aufgenommen werden (sogenannte ambulant-sensitive Krankenhausfälle), nimmt mit abnehmender Facharztdichte ebenso wie mit steigender Krankenhausbettendichte zu (Albrecht 2015).

\subsubsection{Ambulante Notfallversorgung im Krankenhaus}

Eine Zeitreihe von 2010 bis 2015 zeigt, dass sich bei den ambulant behandelten Notfällen die Inanspruchnahme vom ärztlichen Bereitschaftsdienst zu den Notaufnahmen verschoben hat (s. Abb. 2): Während 2010 mit 53\% noch mehr als die Hälfte der ambulanten Notfälle je 1.00o GKV-Versicherte vom ärztlichen Bereitschaftsdienst versorgt wurden, so glich sich dies 2012 aus und kehrte sich bis 2015 auf 47\% zu 53\% um. Der größte absolute Zuwachs bei den Notaufnahmen fand von 2012 auf 2013 statt.

Untersucht man die ambulanten Notfälle in den Notaufnahmen je 1.000 GKV-Versicherte nach Kassenärztlicher Vereinigung, so zeigt sich bei Daten des Zentralinstituts für die kassenärztliche Versorgung (Zi): Während die KV Berlin mit ca. 210 pro 1.00o GKV-Versicherten an oberster Stelle steht (mit Abstand gefolgt von der KV Nordrhein, der KV Saarland und der KV WestfalenLippe), verzeichnet die KV Brandenburg weniger als 50 solcher Fälle je 1.000

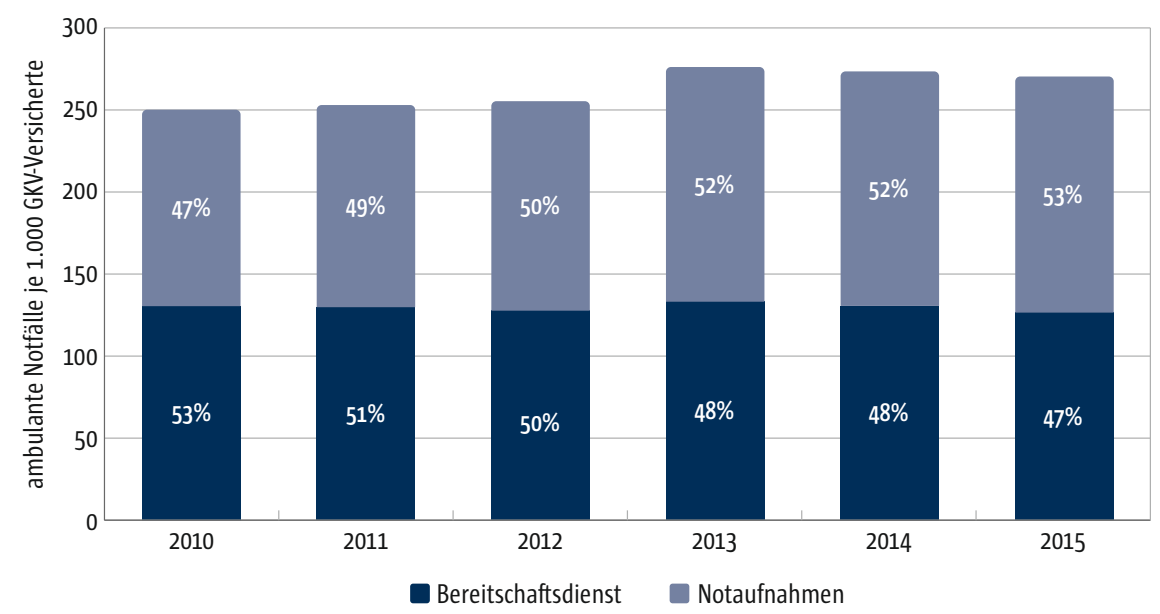

Abb. 2 Ambulante Notfälle je 1.000 GKV-Versicherte. Ohne Bremen, Hamburg, Hessen, Rheinland-Pfalz und Schleswig-Holstein (modifiziert nach SVR 2018 auf Basis von BMG 2017 und Daten des Zi). 


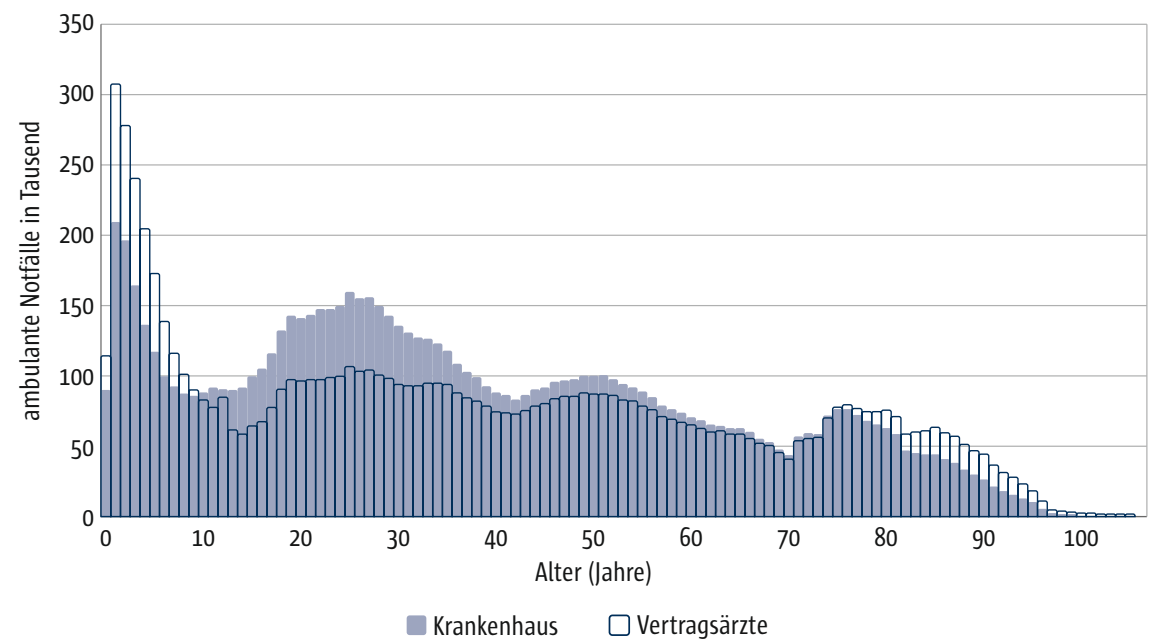

Abb. 3 Ambulante Notfälle bei Vertragsärzten und im Krankenhaus nach Alter und Behandlungsort im Jahr 2015. Ohne Bremen, Hamburg, Hessen und Rheinland-Pfalz (modifiziert nach SVR 2018 auf Basis von Daten des Zi).

GKV-Versicherte (SVR 2018). Hier zeigen sich erhebliche Auswirkungen regionaler Gegebenheiten in ländlichen gegenüber urbanen Räumen. Es ergibt sich ein Durchschnitt von 118 Notfällen je 1.00o GKV-Versicherte.

Unter Qualitätsgesichtspunkten ist besonders die Zahl von (ambulanten) Notfallbehandlungen je Notaufnahme relevant. Laut Analysen des Zi wurden im Jahr 2015 deutschlandweit durchschnittlich 1,7 Patienten pro Stunde (hiervon etwa o,84 Patienten ambulant) in den Notaufnahmen behandelt. Hierbei zeigt sich eine große Spanne zwischen der Auslastung der einzelnen Notaufnahmen: Behandelt ein Viertel der Notaufnahmen durchschnittlich mehr als drei ambulante Notfälle pro Stunde, wird im unteren Viertel nur ein Notfall pro zwei bis drei Stunden versorgt. Die untersten 10\% der Notaufnahmen behandeln im Schnitt sogar nur o,o2 ambulante Notfälle pro Stunde und nehmen somit allenfalls sporadisch an der ambulanten Notfallversorgung teil (Stillfried et al. 2017). Manche Analysen zeigen, dass in Regionen mit hoher sozioökonomischer Deprivation (gemäß sozioökonomischem Gesundheitsindex „SCX“ nach Schulz et al. 2016) weniger häufig ambulante Notfälle versorgt werden, während in den Städten die Inanspruchnahme höher ist und eine höhere jährliche Steigerungsrate aufweist. Kurz gesagt: Je gesünder, urbaner und je weniger sozial belastet die Bevölkerungsgruppe ist, desto eher wird die ambulante Notfallversorgung in Anspruch genommen (Wahlster et al. 2019).

Überdies ist das Notfallaufkommen abhängig vom Alter des Patienten. Untersucht man die ambulanten Notfälle nach Behandlungsort, zeigt sich, dass vor allem Kinder unter zehn Jahren im ärztlichen Bereitschaftsdienst der Niedergelassenen versorgt werden, wohingegen insbesondere junge Erwachsene sich mehr an die Notaufnahmen der Krankenhäuser wenden (s. Abb. 3). Die Gruppe 
der 20- bis 34-Jährigen zeigt im Vergleich zu anderen Altersgruppen einen überproportionalen Anstieg der Inanspruchnahme der ambulanten Notfallversorgung im Krankenhaus (zwischen Ende der Praxisgebühr 2013 und 2015), wobei sie jedoch nur ein geringes Risiko hat, stationär aufgenommen zu werden (Dräther u. Schäfer 2017; Wahlster et al. 2019). Mit zunehmendem Lebensalter - ab ca. 70 Jahren - zeigt sich hingegen ein deutlich ansteigender Anteil der Notfälle, welche stationär aufgenommen werden. Auch der Aufwand der Notfallbehandlung ist altersabhängig und steigt mit zunehmendem Alter.

Im Vergleich zur ambulanten Notfallbehandlung der niedergelassenen Ärztinnen und Ärzte unterscheiden sich ambulante Notfälle im Krankenhaus im Hinblick sowohl auf das Indikationsspektrum als auch auf die erbrachten Leistungen. So wird bildgebende Diagnostik (Röntgen, Ultraschall, CT) im Rahmen der ambulanten Notfallbehandlung fast ausschließlich in Kliniken erbracht. Insgesamt wird bei zwei Dritteln der ambulanten Notfälle in der Notaufnahme eine weitere medizinische Leistung neben der Konsultation (Notfallpauschale) abgerechnet - gegenüber nur einem Zehntel der Patienten im ärztlichen Bereitschaftsdienst (Dräther u. Schäfer 2017).

Während im ambulanten Notfalldienst der niedergelassenen Ärzte ein heterogenes Spektrum an Indikationen behandelt wird, erhalten die Fälle, die ambulant in Notaufnahmen behandelt werden, zu über 50\% Diagnosen aus zwei ICD-Kapiteln: zu einem Großteil „Verletzungen, Vergiftungen und bestimmte andere Folgen äußerer Ursachen" (ICD-10-Kapitel S und T, 43\% im Jahre 2015) sowie „Symptome und abnorme klinische und Laborbefunde“ (ICD-10-Kapitel R, 13\%). Gerade unter das ICD-10-Kapitel R fallen beschreibende Diagnosen aus einem weiten Spektrum - von Kopfschmerzen über Herzrasen bis hin zu Sprachstörungen. Häufig ist auch eine Vorstellung aufgrund von Erkrankungen des Muskel-Skelett-Systems (ICD-10-Kapitel M, 10\%). Krankheiten des Atmungssystems, die bei den Notfällen des ärztlichen Bereitschaftsdiensts die größte Rolle spielen, machen nur 5\% aus (SVR 2018).

Dass Diagnosen aus dem Kapitel R in der ambulanten Notfallversorgung im Krankenhaus einen großen Stellenwert haben, lässt sich aus der klinischen Praxis heraus gut nachvollziehen. Schließlich ist eine der Hauptaufgaben der Notaufnahme, die Notwendigkeit einer stationären Weiterbehandlung zu beurteilen. Während sich der Patient selbst zunächst mit Symptomen vorstellt z.B. Luftnot oder Sprachstörung -, obliegt es dem medizinischen Personal, eine akut lebensbedrohliche bzw. stationär behandlungsbedürftige Ursache, etwa einen Herzinfarkt oder Schlaganfall, auszuschließen. Sollte sich aus der Diagnostik keine Notwendigkeit einer notfallmäßigen stationären Behandlung ergeben, kann der Patient mit einer deskriptiven Diagnose in die ambulanten Strukturen entlassen werden.

Dies erschwert jedoch auch, die Angemessenheit der Notfallinanspruchnahme sowie des Ressourcenverbrauchs in der Notaufnahme objektiv zu beurteilen. Während Datenanalysen sich vor allem auf die Entlassdiagnosen stützen, ist es methodisch schwierig, hierdurch Aussagen über die Leitsymptome und 
Dringlichkeitseinschätzung zu treffen, die bei initialer Präsentation in der Notaufnahme vorlagen. Diese sind jedoch zunächst ausschlaggebend für die nachfolgende Diagnostik und die notfallmäßig erbrachten Leistungen.

\subsection{Patienten mit weniger schwerwiegendem Behandlungsbedarf}

Verschiedene Studien deuten darauf hin, dass ein nicht unerheblicher Anteil der Patientinnen und Patienten in Notaufnahmen auch im Rahmen ambulanter Strukturen behandelt werden könnten. Darauf deutet beispielsweise der teilweise hohe Anteil an Patienten hin, die in der Notaufnahme ambulant behandelt werden. Bei Analysen in München aus den Jahren 2013 und 2014, welche mehr als $96 \%$ des dortigen Notfallaufkommens abdecken, wurden insgesamt zwei Drittel aller Notfallpatienten ambulant behandelt (Trentzsch et al. 2020). Überdies wird bei etwa einem Drittel der ambulant in der Notaufnahme behandelten Patienten nur die Notfall-/Konsultationspauschale ohne weitere Leistung abgerechnet (Haas et al. 2015). Dies ist ein Hinweis darauf, dass die Versorgung teilweise auch durch den ärztlichen Bereitschaftsdienst hätte erfolgen können, der über weniger technische Möglichkeiten als die Notaufnahmen verfügt.

Werden sich selbstständig in der Notaufnahme vorstellende, ambulant behandelte Patienten mit nicht dringlichem Behandlungsbedarf befragt, zeigt sich, dass sich die Selbsteinschätzung der Patienten bezüglich ihrer Dringlichkeit deutlich von der ärztlichen Einschätzung unterscheiden kann: In einer Heidelberger Studie hielten sich 86\% der Patienten für dringlich behandlungsbedürftig, während dies aus ärztlicher Sicht nur für 16\% der Patienten zutraf (Harenberg et al. 2019). Überdies bestehen in anderen Befragungen bei fast einem Drittel der Patienten die Beschwerden seit mehr als drei Tagen und fallen somit auch subjektiv nicht unter einen dringlichen Behandlungsbedarf (Scherer et al. 2017). Gründe, sich dennoch in der Notaufnahme vorzustellen, sind unter anderem erwartete Behandlungsvorteile.

Gründe für die Inanspruchnahme der ambulanten Notfallversorgung im Krankenhaus

Von Patienten berichtete Gründe bei selbstständiger Vorstellung und nichtdringlicher Behandlungsindikation (Somasundaram et al. 2018; Scherer et al. 2017):

- (erwartete) Wartezeiten bzw. mangelnde Verfügbarkeit niedergelassener Ärzte

- Unkenntnis ambulanter Notfallstrukturen (des ärztlichen Bereitschaftsdienstes)

- Empfehlung bzw. Einweisung eines niedergelassenen Arztes

- erwartete Behandlungsvorteile (breites Leistungsspektrum, verfügbare Diagnostik wie Röntgen, CT) 


\subsection{Schwerwiegende Notfälle und ihre Steuerung}

Die Untersuchungen aus München zeigen, dass weniger als ein Fünftel (18\%) aller dort vorstelligen erwachsenen Patienten als zeitkritische Notfälle eingestuft wurden. Von solchen zeitkritischen Patienten wurden $86 \%$ mit dem Rettungsdienst in die Notaufnahme gebracht. Die Hälfte der Patienten in der Notaufnahme benötigte hingegen keine dringende Behandlung (Trentzsch et al. 2020).

Ein erheblicher Teil der Patienten in Notaufnahmen wird allerdings auch stationär aufgenommen, und bei diesen haben Erkrankungen des Kreislaufsystems und Verletzungen mit je ca. 18\% den größten Anteil (Wahlster et al. 2019). Unter diese Kategorien fallen auch mehrere Krankheitsbilder, die seitens einer Konsensusgruppe medizinischer Fachgesellschaften in einem Eckpunktepapier als zeitkritische Krankheitsbilder definiert wurden (Fischer et al. 2016). Eine umgekehrte Betrachtungsweise zeigt: Diagnosen aus den ICD-10-Kapiteln „Neubildungen“ und „Krankheiten des Herz-Kreislauf-Systems“ führen mit größter Wahrscheinlichkeit zu einer stationären Aufnahme - mit ca. 69\% bzw. 65\%. Erkrankungen aus dem ICD-10-Kapitel „Verletzungen“, aus welchem insgesamt in der ambulanten und stationären Versorgung im Krankenhaus am häufigsten kodiert wird, führen hingegen nur in 14,8\% der Fälle zu einer stationären Aufnahme.

Um auf Notfallversorgungsstrukturen mit einer hohen Qualität hinzuwirken, wurden im Jahr 2018 bundesweite Mindeststandards für die Teilnahme an der Notfallversorgung definiert, auf deren Basis Zu- bzw. Abschläge zur Vergütung zur Anwendung kommen. Die Zuordnung zu dem dreistufigen System orientiert sich an strukturellen und prozeduralen Mindestvorgaben. Auch die notfallmedizinischen Fachgesellschaften fordern im o.g. Eckpunktepapier einen einheitlichen Kriterienkatalog für bestimmte zeitkritische Notfälle (Schlaganfall, ST-Hebungsinfarkt, Reanimation bei plötzlichem Kreislaufstillstand, schweres Schädel-Hirn-Trauma, Schwerverletzung/Polytrauma und Sepsis). Dieser soll vorgeben, welche strukturellen und personellen Voraussetzungen ein Krankenhaus vorhalten muss, um zur leitliniengerechten Versorgung jener Krankheitsbilder geeignet zu sein (Fischer et al. 2016).

Schätzungen des Gemeinsamen Bundesausschusses (G-BA) zufolge werden etwa $64 \%$ der allgemeinen Krankenhäuser, die jedoch rund $95 \%$ aller Notfälle behandeln, die Mindestvorgaben zur Teilnahme an der Notfallversorgung erfüllen. Krankenhäuser, die diese Voraussetzungen nicht erfüllen, können im Einzelfall jedoch ebenfalls an der Notfallversorgung teilnehmen (ICES 2018). Die Zahlen deuten im Übrigen darauf hin, dass ein Drittel der Krankenhäuser zusammengenommen nur einen geringen Teil (5\%) der Notfallversorgung übernehmen. Die entsprechend niedrigen Fallzahlen können Auswirkungen auf die Qualität der erbrachten Leistungen haben. 


\section{Stufenmodell des G-BA zur stationären Versorgung von Notfällen}

Hauptkriterien für die drei Stufen sind die Fachabteilungen, das vorzuhaltende Fachpersonal, Intensivkapazitäten und der Umfang der Bereitstellung von Leistungen (G-BA 2018). Es müssen ein angestellter Facharzt je Fachabteilung sowie jeweils ein Facharzt für Innere Medizin, Chirurgie und Anästhesie 24/7 innerhalb von 30 Minuten am Patienten verfügbar sein. Es werden auch spezielle Angebote wie Traumazentren und die Kindernotfallversorgung berücksichtigt.

- Basisnotfallversorgung (Stufe 1): zentrale Notaufnahme mit System zur strukturierten Behandlungspriorisierung innerhalb von 10 Minuten; mindestens Abteilungen für Innere Medizin und Chirurgie; Intensivstation mit mindestens 6 Betten (davon 3 Beatmungsbetten); CT 24/7 verfügbar

- Erweiterte Notfallversorgung (Stufe 2): neben den Anforderungen der Stufe 1 mindestens zwei weitere Fachabteilungen bestimmter Kategorie; MRT; Primärdiagnostik des Schlaganfalls mit Möglichkeit zur Einleitung einer Initialtherapie; notfallendoskopische Intervention am oberen Gastrointestinaltrakt; therapeutischer Herzkatheter; 10 Beatmungsbetten; Beobachtungsstation mit mindestens 6 Betten; Hubschrauberlandestelle

- Umfassende Notfallversorgung (Stufe 3): neben den Anforderungen der Stufe 2 mindestens drei weitere Fachabteilungen bestimmter Kategorie; 20 Beatmungsbetten; Hubschrauberlandestelle ohne Zwischentransport

Neben den Stufen der Notaufnahmen gibt es spezialisierte Angebote zur Versorgung von Schwerverletzten (Traumazentren) sowie von Patienten mit Schlaganfällen oder Herzinfarkten. Gerade für zeitkritische Krankheitsbilder wird das Outcome, also das Überleben bzw. die spätere Lebensqualität des Patienten, nicht nur von der Dauer bis zur ersten stationären Therapiemaßnahme, also der Erreichbarkeit des nächstgelegenen Krankenhauses, sondern auch von der Verfügbarkeit einer definitiven Kausaltherapie (zum Beispiel Herzkatheter) im versorgenden Krankenhaus beeinflusst. So lässt sich beispielhaft an der Notfallversorgung von Herzinfarktpatienten zeigen, dass in Tageszeiten, in denen wegen geringeren erwarteten Patientenaufkommens weniger personelle Ressourcen vorgehalten werden (Nacht und Wochenende), ein wichtiger Qualitätsparameter, nämlich die Zeit vom Eintreffen des Patienten bis zum Beginn derTherapie, verlängert ist und in der Folge auch die Sterblichkeit steigt (Sorita et al. 2014). Die Zeit verlängert sich zusätzlich, wenn Patienten (zunächst) in ein Krankenhaus ohne Herzkathetermöglichkeit eingeliefert werden.

Analysen des Zi legen nahe, dass der Zusammenhang zwischen durchschnittlicher Auslastung der Notaufnahme und dem Behandlungsergebnis auch für andere lebensbedrohliche Notfälle wie Schlaganfall, akutes Nierenversagen oder Sepsis gilt: In größeren Notaufnahmen mit einer höheren behandelten Patientenzahl (mehr als 2,3 Patienten pro Stunde) ist die Überlebenswahrscheinlichkeit um 30\% bis 50\% höher als in kleinen (Stillfried et al. 2017). Nicht 
wenige Experten leiten daraus die Empfehlung ab, aus Qualitätsgründen den Stellenwert spezialisierter Notaufnahmen mit hohen Fallzahlen sowie denjenigen von spezifischen Notfallzentren besonders zu fördern.

\subsubsection{Daten und Qualitätstransparenz}

Für die Qualitätsmessung und -verbesserung in der (stationären) Notfallversorgung sind umfassende und vergleichbare Daten eine wichtige Voraussetzung. Im Bereich der Notfallversorgung gibt es zwar einzelne Softwarelösungen z.B. für die Übertragung vom Rettungsdienst an ein Krankenhaus. Es sind bisher aber keine entsprechenden standardisierten und vollständigen Datenflüsse und Dokumentationsstrukturen etabliert, insbesondere nicht einrichtungs- und sektorenübergreifend. Internationale Beispiele für eine fortgeschrittene Digitalisierung und Vernetzung der Patientenakten gibt es beispielsweise in Dänemark. Der Nutzen könnte bereits in der Wahl der richtigen Versorgungsebene und Zieleinrichtung liegen, aber auch in der nachträglichen Qualitätstransparenz. Auch Elemente des Risikomanagements wie „Critical Incident Reporting Systems“ “(CIRS) und Teamtrainings können hier eine wichtige Rolle spielen.

\section{Take home messages}

- Die Inanspruchnahme der Notaufnahmen von Krankenhäusern steigt in den letzten Jahren.

- Die Zunahme der Inanspruchnahme ambulanter Notfallbehandlung im Krankenhaus ist besonders bei Jüngeren (20-bis 34-Jährige) gestiegen. Bei Älteren ist hingegen die Wahrscheinlichkeit, stationär aufgenommen zu werden, höher.

- Die häufigsten Diagnosen bei der ambulanten Notfallbehandlung im Krankenhaus sind Verletzungen und Vergiftungen. Das Spektrum unterscheidet sich vom ärztlichen Bereitschaftsdienst. Stationär aufgenommen werden Patienten häufig aufgrund von Herz-KreislaufErkrankungen.

- Bei Notfallpatienten mit zeitkritischen Diagnosen zeigt sich ein besseres Outcome (z.B. Überleben), wenn die Behandlung in größeren Notaufnahmen bzw. spezialisierten Zentren erfolgt.

\section{Literatur}

Albrecht M (2015) Ambulantes Potential in der stationären Notfallversorgung. Ergebnisbericht zur Projektphase I für das Zentralinstitut für die kassenärztliche Versorgung in Deutschland. IGES Institut.

Behringer W, Buergi U, Christ M, Dodt C, Hogan B (2013) Fünf Thesen zur Weiterentwicklung der Notfallmedizin in Deutschland, Österreich und der Schweiz. Notfall Rettungsmed 16(8): 625-626.

Berchet C (2015) Emergency care services: trends, drivers and interventions to manage the demand. OECD Health Working Papers (83). 
BMG - Bundesministerium für Gesundheit (2017) Mitglieder und Versicherte der GKV. KM6.

Carter E, Pouch S, Larson E (2014) The Relationship Between Emergency Department Crowding and Patient Outcomes: A Systematic Review. J Nurs Scholarsh 46(2): 106-115.

Destatis/Statistisches Bundesamt (2019) Fallpauschalenbezogene Krankenhausstatistik.

Dräther H, Schäfer T (2017) Die ambulante Notfallversorgung in Notfallambulanzen und bei Vertragsärzten. In: Klauber I, Geraedts M, Friedrich |, Wasem I (Hrsg.): Krankenhaus-Report 2017. Schwerpunkt: Zukunft gestalten. Schattauer Verlag, Stuttgart.

Fischer M, Kehrberger E, Marung H, Moecke H, Prückner S, Trentzsch H, Urban B (2016) Eckpunktepapier 2016 zur notfallmedizinischen Versorgung der Bevölkerung in der Prähospitalphase und in der Klinik. Notfall Rettungsmed 19: 387-395.

G-BA - Gemeinsamer Bundesausschuss (2018) Beschluss über die Erstfassung der Regelungen zu einem gestuften System von Notfallstrukturen in Krankenhäusern gemäß $\$ 136 c$ Abs. 4 SGB V. URL: https://www.g-ba. de/downloads/39-261-3301/2018-04-19_Not-Kra-R_Erstfassung.pdf (abgerufen am 02.06.2020)

Geissler A, Quentin W, Busse R (2017) Umgestaltung der Notfallversorgung. In: Klauber I, Geraedts M, Friedrich J, Wasem I (Hrsg.): Krankenhaus-Report 2017. Schwerpunkt: Zukunft gestalten. Schattauer Verlag, Stuttgart: 41-59.

Haas C, Larbig M, Schöpke T, Lübke-Naberhaus KD, Schmidt C, Brachmann M, Dodt C (2015) Gutachten zur ambulanten Notfallversorgung in Krankenhäusern - Fallkostenkalkulation und Strukturanalyse. URL: https://www. dkgev.de/fileadmin/default/Mediapool/2_Themen/2.2_Finanzierung_und_Leistungskataloge/2.2.4._Ambulante_Verguetung/2.2.4.4._Ambulante_Notfallvehandlung_durch_Krankenhaeuser/2015-02-17_Gutachten_zur_ambulanten_Notfallversorgung_im_Krankenhaus_2015.pdf (abgerufen am 10.07.2020).

Harenberg L, Oßwald HM, Jaschonek H, Nagel S (2019) Selbsteinschätzung der Behandlungsdringlichkeit bei Vorstellung in einer neurologischen Notfallambulanz. Ergebnisse einer Patientenbefragung. Nervenarzt 90: 175 .

IGES (2018) Folgenabschätzung einer gestuften Notfallversorgung. IGES Institut, Berlin. URL: https://www.iges. com/sites/iges.de/myzms/content/e6/e1621/e10211/e22175/e23180/e23181/e23184/attr_objs23190/ IGES_Folgenabschaeztzung_Notallversorgung_072018_ger.pdf (abgerufen am 10.07.2020).

Morley C, Unwin M, Peterson G, Stankovich I, Kinsman L (2018) Emergency department crowding: A systematic review of causes, consequences and solutions. PLOS ONE 13(8): e020331.

Scherer M, Lühmann D, Kazek A, Hansen H, Schäfer I (2017) Patienten in Notfallambulanzen. Dtsch Arztebl Int 114(39): 645-652.

Schreyögg I, Busse R, Bäuml M, Krämer |, Dette T, Geissler A (2014) Forschungsauftrag zur Mengenentwicklung nach $\S 17$ b Abs. 9 Khg. Endbericht.

Schulz M, Czihal T, Erhart M, Stillfried D (2016) Korrelation zwischen räumlichen Sozialstrukturfaktoren und Indikatoren des medizinischen Versorgungsbedarfs. Gesundheitswesen 78: 290-297.

Somasundaram R, Geissler A, Leidel BA, Wrede C (2018) Beweggründe für die Inanspruchnahme von Notaufnahmen. Ergebnisse einer Patientenbefragung. Gesundheitswesen 80(07): 621-627.

Sorita A, Ahmed A, Starr SR, Thompson KM, Reed DA, Prokop L, Shah SD, Murad MH, Ting HH (2014) Off-hour presentation and outcomes in patients with acute myocardial infarction: systematic review and metaanalysis. BMI 348: f7393.

Spindler I (2020) Fallpauschalenbezogene Krankenhausstatistik: Diagnosen und Prozeduren der Krankenhauspatienten auf Basis der Daten nach $\S 21$ Krankenhausentgeltgesetz. In: Klauber I, Geraedts M, Friedrich I, Wasem I und Beivers A (2020): Krankenhaus-Report 2020. Springer Verlag, Berlin.

Stillfried von D, Czihal T, Erhart M (2017) Rolle der Krankenhäuser in der Notfallversorgung in Deutschland: Daten belegen massiven Reformbedarf. Zi-Paper 11. URL: https://www.zi.de/fileadmin/images/content/ Publikationen/Zi-Paper_11-2017_Notfallversorgung.pdf (abgerufen am 02.06.2020)

SVR - Sachverständigenrat zur Begutachtung der Entwicklung im Gesundheitswesen (2018) Bedarfsgerechte Steuerung der Gesundheitsversorgung. Medizinisch Wissenschaftliche Verlagsgesellschaft, Berlin.

Trentzsch H, Dodt C, Gehring C, Veser A, Jauch K-W, Prückner S (2020) Analyse der Behandlungszahlen in den Münchener Notaufnahmen des Jahres 2013/2014. Gesundheitswesen 82: 431-440.

Wahlster P, Czihal T, Gibis B, Henschke C (2019) Sektorenübergreifende Entwicklungen in der Notfallversorgung. Gesundheitswesen. URL: DOI: 10.1055/a-0820-3904. 


\section{Versorgungssystem unter Qualitätsaspekten gestalten}

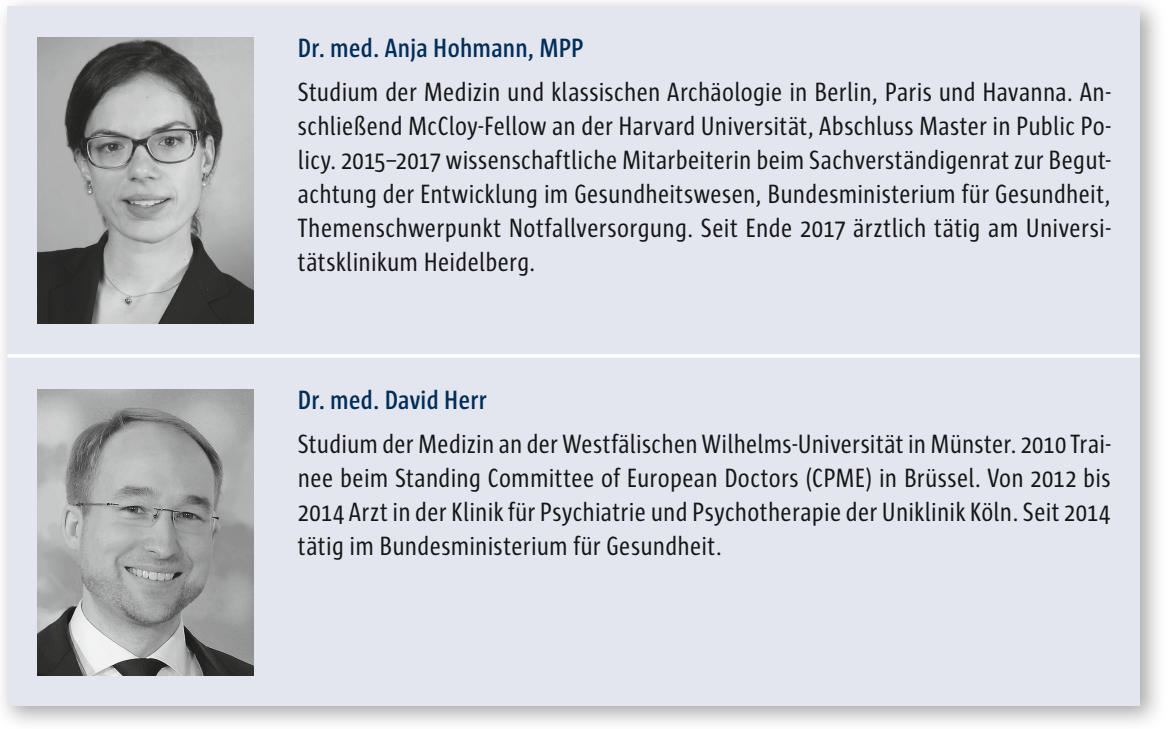

